\title{
The heliospheric magnetic field over the Hale cycle
}

Article

Published Version

Schwadron, N. A., Owens, M. and Crooker, N. U. (2008) The heliospheric magnetic field over the Hale cycle. Astrophysics and Space Sciences Transactions, 4 (1). pp. 19-26. ISSN 1810-6536 doi: https://doi.org/10.5194/astra-4-19-2008 Available at https://centaur.reading.ac.uk/5826/

It is advisable to refer to the publisher's version if you intend to cite from the work. See Guidance on citing.

To link to this article DOI: http://dx.doi.org/10.5194/astra-4-19-2008

Publisher: Copernicus

All outputs in CentAUR are protected by Intellectual Property Rights law, including copyright law. Copyright and IPR is retained by the creators or other copyright holders. Terms and conditions for use of this material are defined in the End User Agreement.

\section{www.reading.ac.uk/centaur}

\section{CentAUR}

Central Archive at the University of Reading

Reading's research outputs online 


\title{
The Heliospheric Magnetic Field over the Hale Cycle
}

\author{
N. A. Schwadron, M. Owens, and N. U. Crooker \\ Boston University, Center for Space Physics, Center for Integrated Spaceweather Modeling, 725 Commonwealth Ave, Boston \\ MA, 02215, USA
}

Received: 25 September 2007 - Revised: 20 December 2007 - Accepted: 17 January 2008 - Published: 30 May 2008

\begin{abstract}
The concept that open magnetic flux of the Sun (rooted with one and only one footpoint at the Sun) is a conserved quantity is taking root in the heliospheric community. Observations show that the Sun's open magnetic flux returns to the baseline from one solar minimum to the next. The temporary enhancement in the $1 \mathrm{AU}$ heliospheric magnetic flux near solar maximum can be accounted for by the temporary creation of closed magnetic flux (with two footpoints at the Sun) during the ejection of coronal mass ejections (CMEs), which are more frequent near solar maximum. As a part of the International Heliophysical Year activities, this paper reviews two recently discussed consequences of open flux conservation: the reversal of open magnetic flux over the solar cycle driven by Coronal Mass Ejections and the impacts of open flux conservation on the global structure of the heliospheric magnetic field. These studies demonstrate the inherent linkages between coronal mass ejections, footpoint motions back at the Sun, and the global structure and evolution of the heliospheric magnetic field.
\end{abstract}

\section{Introduction}

The sun's differential rotation and the related internal solar dynamo result in the Sun's solar cycle evolution (Babcock, 1961; Leighton, 1969). Magnetic flux emerges at midlatitudes carrying the helicity generated by the differential shear over the previous solar cycle. This polarity of the emerging field opposes the dominant polarity of large-scale solar magnetic field. Eventually, the emergence of new magnetic flux brings about the polarity reversal of the large-scale solar magnetic field (e.g. Schrijver et al., 2002; Wang et al., 2002, and references therein).

Correspondence to: N. A. Schwadron (nathanas@bu.edu)
The heliosphere's open magnetic field also undergoes the reversal seen in the large-scale solar magnetic field. However, the solar magnetic field reversal and the heliospheric magnetic field reversal appear manifestly different. The solar magnetic field reversal occurs through a complex process where the polar coronal holes are broken up in the rising phase of the solar cycle and smaller coronal holes appear at all latitudes during solar maximum. In the declining phase of solar activity, coronal holes are gradually assembled near the poles, although their dominant polarity opposes that of the previous solar minimum. In contrast, the heliosphere's magnetic field reversal is much more organized, appearing almost as a large-scale rotation (Smith et al., 2000; Smith and Balogh, 2003; Jones et al., 2003).

Important questions are how the reversal of the solar magnetic field controls the Sun's open magnetic field, and what are the global impacts of differential rotation on the heliospheric magnetic field. As a part of International Heliophysical Year activities, this paper reviews recent work showing the interplay between solar dynamics (differential rotation, and CMEs) and the structure and evolution of the open magnetic flux of the heliosphere.

As detailed in Sect. 2, CMEs may play an important role in causing the open field reversal. The interchange reconnection process envisioned by Fisk and Schwadron (2001) involved the interaction of coronal loops and open field lines. This is similar in some respects to the interactions between open field lines and the very large scale closed loops drawn out by CMEs. The open field lines eventually interchange reconnect with the large CME loops, causing large displacements in the footpoints of open field lines.

In Sect. 3, we discuss the impacts of footpoint motion on the Sun for the large-scale structure of the heliospheric magnetic field. In particular, during most of the solar cycle ( $\sim 9$ of 11 years) when the Sun has large polar coronal holes, footpoint motions lead to magnetic connections of the open magnetic flux between low latitudes (with slow wind) and

Published by Copernicus Publications on behalf of the Arbeitsgemeinschaft Extraterrestrische Forschung e.V. 
high latitudes (with fast wind). The latitudinal transitions between slow and fast wind (between low and high latitudes) form interaction bands in the outer heliosphere and generate a distorted configuration in the open field (called 'FALTS'). The impact of this large-scale magnetic structure on energetic particles and cosmic rays is not yet understood.

\section{The CME model for field reversal}

Beyond the Alfvén point $\left(\sim 15 R_{\odot}\right)$, the supersonic solar wind drags out its embedded magnetic field, and information cannot flow back to the Sun. Therefore, kinks, braids, turbulence, and all other non-radial field components flow outward, away from the Sun beyond the Alfvén point. Fundamentally then, the reorientation of the Sun's open magnetic field must occur inside the Alfvén point, and then information about the reorientation flows outward with the solar wind.

The traditional answer to the question of how the open field reverses is that the Sun sheds its open flux from one solar minimum to the next and reconstitutes itself with new open flux of the reversed polarity. In a sense, the process affecting open and closed flux would be the same. This is essentially the view espoused by Wang and Sheeley (2003), who used potential field solutions to show how the emerging dipoles cause closed coronal loops to rise and destroy old open magnetic flux or create new open magnetic flux.

A fundamentally different approach to the problem was proposed by Fisk and Schwadron (2001). They argued that open magnetic flux should be considered a conserved quantity. The migration of open magnetic flux, which amounts to a massive rotation of open flux, occurs through the process of media diffusion involving reconnection between open magnetic field lines and a large underlying medium of closed loops. In each reconnection event between an open field line and a closed coronal loop, the footpoint of the open magnetic field line is interchanged with the footpoint on one side of the loop - thus termed interchange reconnection (Crooker et al., 2002). The interchange reconnection process itself conserves the net amount of open magnetic flux. Therefore, in this scenario the Sun does not shed its open magnetic flux from the previous solar cycle. The reversal of the open field should then be thought of as a reconfiguration of the open magnetic flux.

The two views of open field reversal differ fundamentally in one respect. If open magnetic flux is a conserved quantity as suggested by Fisk and Schwadron (2001), then we should see the return to the same interplanetary field strengths from one solar minimum to the next. In fact, heliospheric flux is observed to return to the same value each solar minimum (e.g. Arge et al., 2002), which is direct evidence for open flux conservation over the solar cycle. If the open flux is not conserved throughout the solar cycle, then open-flux creation must exactly balance open-flux destruction (i.e. disconnection), by some unknown mechanism.

One of the trends that appears to contradict open flux conservation is the apparent increase (approximate doubling) of interplanetary field strengths near solar maximum. However, Owens and Crooker (2006) demonstrated that CMEs introduce new closed flux into the heliosphere, which subsequently opens through interchange reconnection over long timescales ( $\sim 40-50$ days). In this scenario open magnetic flux is not destroyed or created. Instead because CMEs are released much more frequently near solar maximum $(\sim 3$ CMEs per day), they raise the level of transient closed flux, which in turn raises the interplanetary field strengths. In the return to solar minimum, the frequency of CMEs drops significantly, and the majority of interplanetary magnetic flux is open flux. Therefore, if open flux is conserved, there is a minimum baseline of interplanetary field strengths that is approached near solar minimum. Near solar maximum, the temporary addition of closed flux by CMEs raises the interplanetary field strengths.

There is observational evidence that CMEs play an important role in the the Sun's open field reversal. Gopalswamy et al. (2003) noted that the cessation of high latitude CMEs corresponded to the end the polar field reversal. Low (2004) suggested that coronal mass ejections remove old open solar flux from the corona, making way for the replacement by new open flux of opposite polarity.

We outline here a model that was recently discussed by Owens et al. (2007) in which CMEs drive the heliospheric magnetic field reversal. The model makes one key assumption. The total open flux of the Sun is conserved over the solar cycle. However, the total flux on a given sphere increases near solar maximum as CMEs temporarily add closed flux, as described by Owens and Crooker (2006). This new closed flux eventually (on a $\sim 40-50$ day timescale) opens via interchange reconnection with open field lines below the Alfvén point.

CME's have a dominant polarity that opposes the average open flux polarity of the large-scale field. Therefore, when the added CME flux opens, the interchange reconnection process moves open magnetic flux toward a new configuration. In particular, the interchange reconnection process moves magnetic flux toward a reversal of the open field polarity. We emphasize that the reversal process does not occur through a single event, but rather through progression of interchange reconnection events associated with and driven by individual CMEs.

Figure 1 illustrates our scenario for the topological evolution of the solar magnetic field as a result of the steps of interchange reconnection between open magnetic flux and CMEs. Horizontal rows of the figure follow in sequence from the top of the page. Light red (blue) shaded regions are negative (positive) polarity coronal holes $(\mathrm{CHs})$, and red (blue) lines are the inward (outward) magnetic field lines. In the top row, the black lines show the magnetic field associ- 

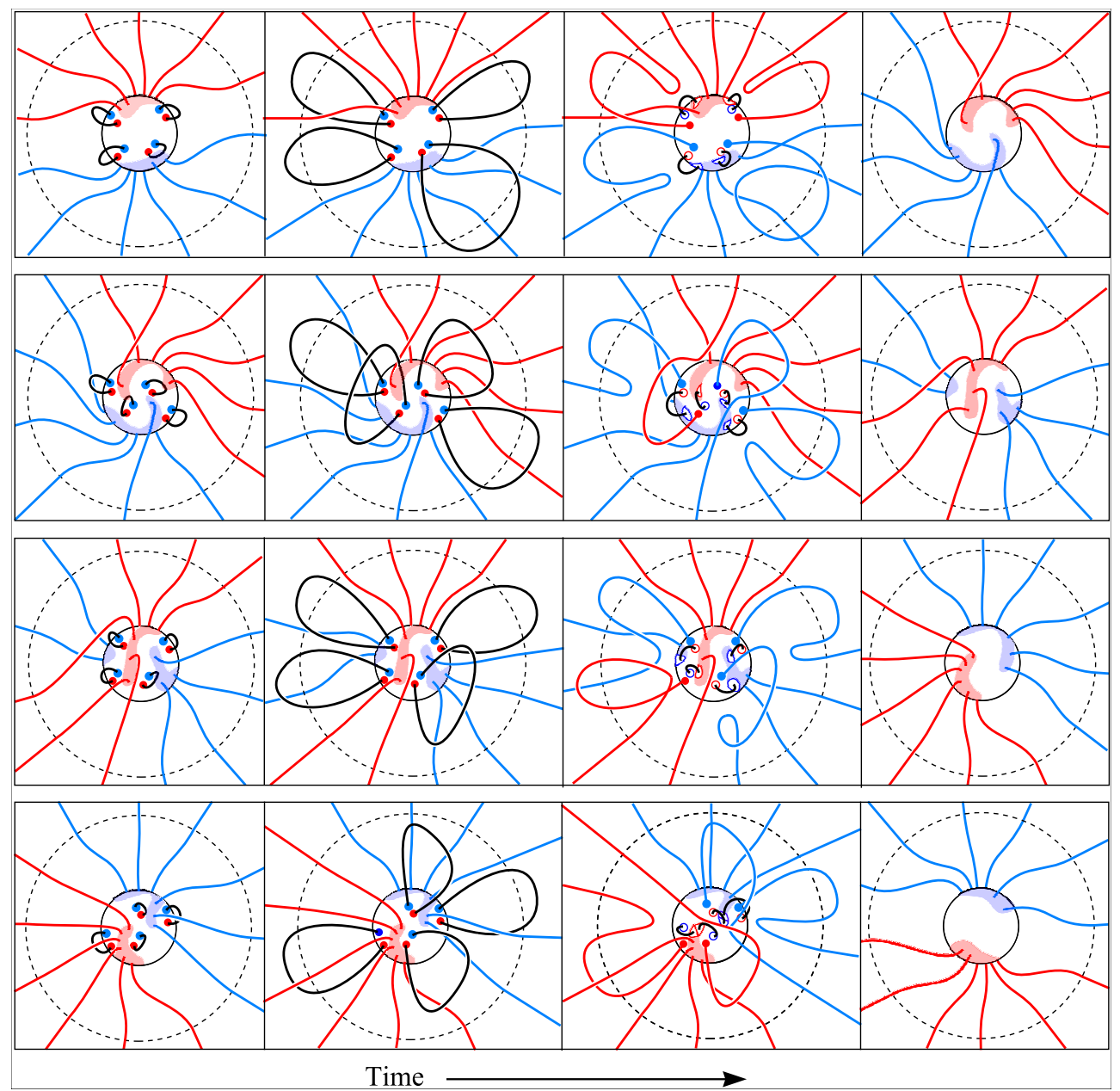

Fig. 1. A sketch of the topological evolution of the solar magnetic field. Black dashed lines show a heliocentric distance past which field lines are considered "open" to the heliosphere. Light red (blue) shaded regions are negative (positive) polarity coronal holes (CHs), whereas red (blue) lines are the associated inward (outward) magnetic field lines. Black lines show the closed CME loops, with the red (blue) circles showing the negative (positive) polarity CME footpoints. Negative (positive) open flux that has interchange reconnected to produce closed loops is shown as white regions with red (blue) outlines. See the main text for description. This figure is from Owens et al. (2007).

ated with a CME. As the CME lifts off, it temporarily adds magnetic flux (second panel from the left). Eventually this magnetic flux interchange reconnects (third panel from left), which then creates latitudinal extensions of the coronal holes (fourth panel from left) due to the forced migration of open magnetic flux from within the coronal hole. We stress the importance of the CME polarity, which opposes the average open field polarity and upon interchange reconnection forces open magnetic flux out of the coronal hole.

The process of open field evolution and migration following interchange reconnection with CMEs continues throughout the solar cycle as illustrated in the subsequent rows of Fig. 1. The first row of the Figure shows the migration in the beginning stages of increased solar activity (the rise phase). In the second row (the pre-reversal phase) the Sun is closer to solar maximum and the continued forcing by CMEinduced interchange reconnection leads to the development of ever greater extensions of coronal hole boundaries. At some point, the organization of polar coronal holes is replaced by a series of coronal holes spread unevenly about the Sun. The third row shows the Sun as it moves past solar maximum (the post-reversal phase) and the continued forcing by CMEs leads to new polar coronal holes with reversed polarity (compared to the previous solar minimum). In the final row (the declining phase), solar activity quiets, the Sun moves into a new solar minimum and completes the large-scale field reversal. Throughout the reversal, the polarity of CMEs on average opposes the open field polarity of the previous solar minimum. The solar cycle shown is analogous to cycle 22, with the start of the cycle characterized by negative open flux from the north pole of the Sun. Following Bothmer and Rust (1997), Bothmer and Schwenn (1998), and Rees and Forsyth (2003), northern (southern) hemisphere CMEs have a negative (positive) leading footpoint, which is positioned closer to 
the equator than the trailing footpoint throughout the whole cycle.

With the open flux conservation assumption, there are two key requirements that must be fulfilled for CMEs to achieve the open field reversal

- CMEs have a dominant polarity that opposes the average open flux polarity of the previous solar minimum. This requirement is consistent with in-ecliptic observations. Bothmer and Rust (1997) and Bothmer and Schwenn (1998) found that the magnetic field polarities of filaments and associated magnetic clouds obey a trend similar to the Hale law for sunspot polarity (Hale and Nicholson, 1925). The polarity of the leading (in the sense of solar rotation), lower latitude sunspot is determined by the dominant hemispheric polarity at the start of the solar cycle. The Hale cycle for CME footpoints is supported by Ulysses observations of magnetic clouds over solar cycle 23 (Rees and Forsyth, 2003). When coupled with the assumption of conserved open flux, repeated CME eruption and subsequent interchange reconnection lead to a net latitudinal transport of open solar flux.

- The second requirement is that the latitudinal separation of CME footpoints is large enough to reverse the open field through repeated CME ejection.

To quantify the second requirement, it is necessary to estimate the magnetic flux carried out by a typical ICME $\left(\phi_{\mathrm{CME}}\right)$, which is loosely constrained observationally (e.g. Lynch et al., 2005). It is instructive to investigate what further limitations can be placed on $\phi_{\mathrm{CME}}$ based on the evolution of the open flux over the solar cycle.

Given open flux conservation, which we have assumed, the change in heliospheric unsigned flux over the solar cycle is given by $\sim 4 \pi \mathrm{R}^{2}|\Delta \mathbf{B}| / \sqrt{2}$, where $R$ is $1 \mathrm{AU}$ and $|\Delta \mathbf{B}|$ is the difference in the magnetic field intensity at 1 AU between solar minimum and maximum, observed to be $\sim 3 \mathrm{nT}$ (e.g. Owens and Crooker, 2006). We also take an average $45^{\circ}$ Parker spiral angle at $1 \mathrm{AU}$. Thus the solar cycle flux increases by $\Delta \Phi \sim 6 \times 10^{14} \mathrm{~Wb}$, which we assume is due to the temporary addition of closed ICME flux. At solar minimum $|\mathbf{B}| \sim 5 \mathrm{nT}$, suggesting that the net open flux is $\Phi_{0} \sim 1 \times 10^{15} \mathrm{~Wb}$ (Smith, 1993; Bieber and Rust, 1995; Smith and Phillips, 1997). Counterstreaming electrons (CSEs) are indicators of closed heliospheric magnetic flux (Gosling et al., 1987). As CSEs are routinely seen within magnetic clouds at $5 \mathrm{AU}$ (e.g. Riley et al., 2004; Crooker et al., 2004), and typical ICME speeds are $\sim 450 \mathrm{~km} / \mathrm{s}$, the minimum time for ICME flux opening must be $\sim 20$ days. For typical CME rates of 3 per day (Yashiro et al., 2004) this allows at least $N_{\mathrm{CME}}=60 \mathrm{CMEs}$ to contribute to the temporary closed flux from ICMEs at any one instant on average. Hence the maximum value of Interplanetary CME (ICME) axial flux is $\phi_{\mathrm{CME}}=\Delta \Phi /\left(2 N_{\mathrm{CME}}\right) \sim 5 \times 10^{12} \mathrm{~Wb}$. Note that an individual ICME contributes twice its flux to the open flux since the closed field lines are tied to the Sun at both ends. Further, a fraction of $\chi_{\mathrm{ix}} \sim 50 \%$ of the CME flux opens shortly after the CME leaves the Sun. The maximum value of axial CME flux $\left(\sim 5 \times 10^{12}\right)$ is in rough agreement with the observational estimate of $\phi_{\mathrm{CME}}=1.5 \times 10^{12} \mathrm{~Wb}$, based on fits to magnetic cloud observations at $1 \mathrm{AU}$ (Lynch et al., 2005) (the average axial CME flux ejected from the Sun is $3 \times 10^{12}$ ). After interchange reconnection with a CME, a quantity of open flux equal, on average, to the axial CME flux $\left(\phi_{\mathrm{CME}} / \chi_{\mathrm{ix}}\right)$ moves by an angular distance equal to the latitudinal separation of the CME footpoints, $\langle\Delta \theta\rangle$. Therefore, the open field reversal requires $N_{\text {cycle }}$ CMEs with Hale's law helicities, where

$N_{\text {cycle }}=\frac{\Phi_{0} \chi_{\mathrm{ix}}}{\phi_{\mathrm{CME}}} \frac{180^{\circ}}{\langle\Delta \theta\rangle}$

Using the maximum estimate of the average CME flux of $\phi_{\mathrm{CME}}=5 \times 10^{13} \mathrm{~Wb}$, with $\Phi_{0}=1 \times 10^{15} \mathrm{~Wb}$, $f_{\mathrm{CME}}=3 \mathrm{day}^{-1}$, and $\chi_{\mathrm{ix}}=50 \%$, the observed solar cycle length of $\tau_{\text {cycle }}=11$ years (where $N_{\text {cycle }}=\tau_{\text {cycle }} f_{\mathrm{CME}}$ ) can be brought about as long as the latitudinal CME footpoint separation is $\langle\Delta \theta\rangle \sim 1.5^{\circ}$. With the magnetic flux estimate of $\phi_{\mathrm{CME}}=1.5 \times 10^{12} \mathrm{~Wb}$ (Lynch et al., 2005), we find a somewhat larger latitudinal separation of CME footpoints of $\langle\Delta \theta\rangle \sim 5^{\circ}$.

\section{The Field Configurations of the Heliosphere's Interaction Bands}

In the previous section, we discussed the evolution of the large-scale heliospheric magnetic field and its reversal as the result of interchange reconnection driven by CMEs. In this section, we focus instead on the the field configurations of the outer heliosphere driven by the smaller scale processes (differential rotation and interchange reconnection with loops) that lead to footpoint motion back at the Sun.

Prior to the introduction of footpoint motion by Fisk (1996), for many years it had been assumed that footpoints of the Sun's open magnetic field lines move bodily with the Sun $^{1}$. This assumption led Parker (1958) to the following expression for the structure of the heliospheric magnetic field:

$\mathbf{B}_{p}=B_{s s}\left(\frac{R_{s s}}{r}\right)^{2}\left(\hat{e}_{r}-\frac{\Omega_{\odot} r \sin \theta}{V} \hat{e}_{\phi}\right)$

Here $\hat{e}_{r}$ is the radial direction, $r$ is the heliocentric radius, $\hat{e}_{\phi}$ is the azimuthal direction, $\theta$ is the co-latitude, $V$ is the solar wind speed, $R_{s s}$ is the radius of the source surface (Schatten et al., 1969) where the solar wind flow becomes entirely ra-

\footnotetext{
${ }^{1}$ Here, open magnetic field lines are defined as the field lines that extend from the Sun out through the heliosphere, and may be differentiated from the Sun's closed structures such as coronal loops. The footpoint of an open magnetic field line is the point of intersection of the solar surface with the field line.
} 


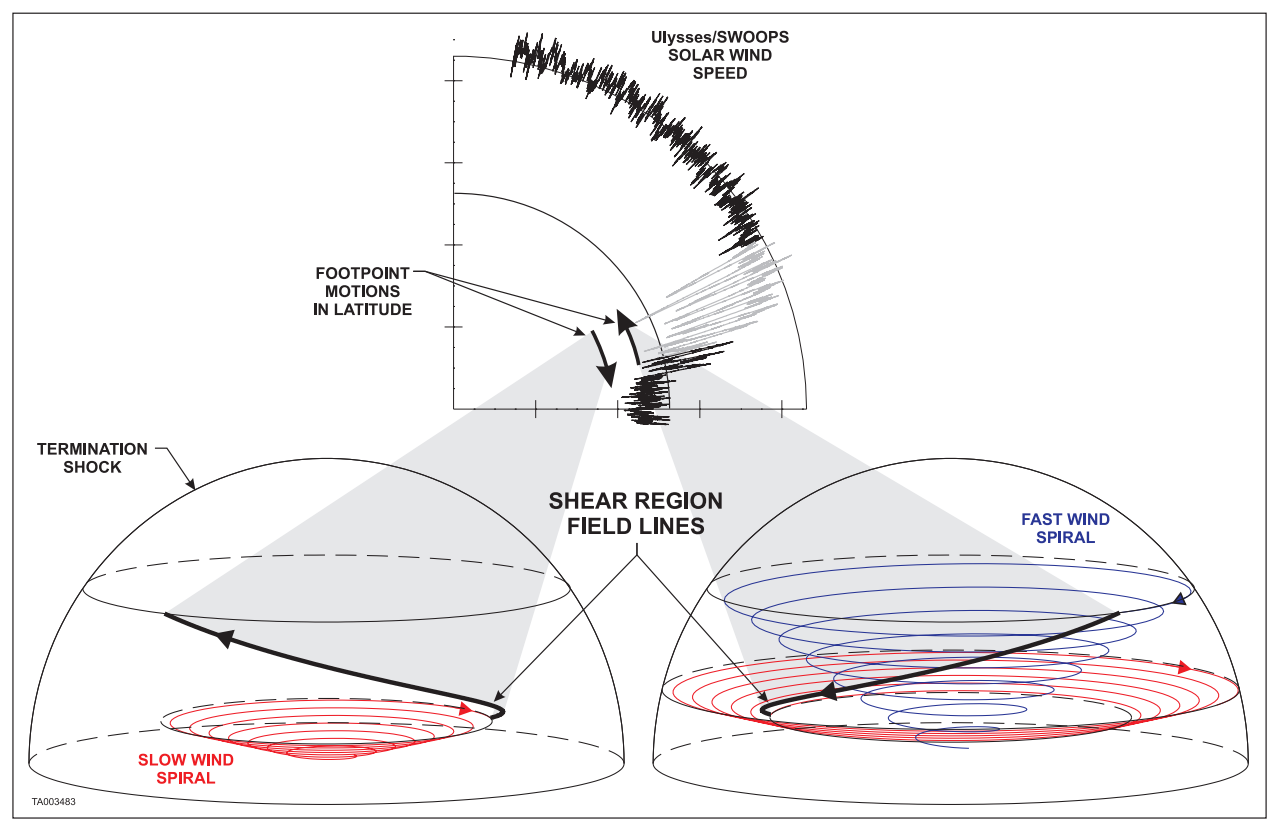

Fig. 2. The three dimensional configurations of the magnetic fields in the distant interaction bands are indicated in the lower panels by black lines. The left (right) lower panel applies for footpoint motions from fast (slow) wind into the slow (fast) solar wind. The streamline in slow wind is indicated by the red curve, and the streamline in fast wind is indicated by the blue curve. The upper panel indicates the sense of footpoint motions and solar wind speeds measured by Ulysses as a function of latitude observed in 1995 and 1996. The field lines were drawn in this figure for $\left|\omega_{\theta}\right|=0.15 \Omega_{s}$, for an interaction region between a latitude $\lambda_{s}=15^{\circ}$ and $\lambda_{f}=35^{\circ}$, and for solar wind speeds between $450 \mathrm{~km} / \mathrm{s}$ and $750 \mathrm{~km} / \mathrm{s}$.

dial, $B_{s s}$ is the field strength at that source surface, and $\Omega_{\odot}$ is the Sun's equatorial rotation rate.

Fisk (1996) pointed out that the Sun's open magnetic field footpoints should not rotate bodily with the Sun. Coronal holes, which are the source regions of fast solar wind, are observed to rotate bodily with the Sun over many solar rotations (e.g. Bird and Edenhofer, 1990), but the parcels of plasma on the solar surface are seen to rotate differentially through the coronal hole region (e.g. Snodgrass, 1983). This led to the concept of magnetic footpoint motion on the Sun (Nash et al., 1988). The footpoints of open magnetic field lines are dragged by the differentially rotating plasma into, through and ultimately out of coronal holes. Fisk et al. (1999) and Fisk and Schwadron (2001) argued that the open magnetic field footpoints of the Sun execute a global circulation pattern where footpoint motions are driven by differential motion within coronal holes and sustained by reconnection between open magnetic field lines and large coronal loops beyond coronal hole boundaries.

Footpoint motions on the Sun coupled with large-scale solar wind speed variations as a function of heliolatitude in the outer heliosphere form highly distorted field structures that have increased radial field components, providing relatively low-speed ions with the ability to move back upstream in the solar wind (Schwadron and McComas, 2003). These "Favored Acceleration Locations at the Termination Shock" (FALTS) shown in Fig. 2 allow for relatively low energy injection, and thus higher efficiency for injecting ions into diffusive shock acceleration at the Termination Shock (TS). We discuss here further implications of these distorted field structures, particularly in the context of recent Voyager $1 \mathrm{ob}-$ servations indicating possible passage beyond the TS (Krimigis et al., 2003).

FALTS are a product of footpoint motions on the Sun and the shearing by the solar wind in a latitudinal band where the tilt of the heliomagnetic axis with respect to the Sun's rotation axis sets up a varying pattern of fast and slow solar wind (Burlaga, 1974; Hundhausen and Burlaga, 1975; Siscoe, 1976; Gosling et al., 1978; Pizzo, 1989). Over much of the solar cycle, excluding periods of very high solar activity, slow solar wind is emitted at low latitudes and fast wind is emitted at higher latitudes. The top central polar plot in Fig. 2 shows the solar wind speed observed by Ulysses as a function of latitude in 1995 and 1996. Interaction regions shown in gray form over the range of heliolatitudes where both fast and slow wind are emitted. As these streams propagate outward, the fast wind overtakes the slow wind, forming compression regions and, typically within 2-3 AU of the Sun, co-rotating shocks; these structures are called co-rotating interaction regions or CIRs. During 1996, for example, interaction regions containing CIRs extended from about $10^{\circ}$ to $30^{\circ}$ latitude (McComas et al., 2000a).

As the CIRs move into the outer heliosphere, the fast and slow streams continue to interact until their speed differences 
wear down, resulting in the formation of large-scale bands of intermediate speeds, referred to here as "interaction bands". The speed in these interaction bands should change relatively smoothly from slow at the low latitude boundary to fast at the high-latitude boundary. This monotonic transition in speed is expected since fast wind is emitted over a larger fraction of the rotation in the higher latitude segments of the interaction band.

For a simple model of the magnetic field in the interaction bands of the outer heliosphere that includes footpoint motions on the Sun, Schwadron and McComas (2003) take a solar wind speed $V$ in the outer heliosphere that has a dependence on co-latitude $\theta, V=V(\theta)$. Consider then the magnetic field in the interaction band that results if footpoint motions on the Sun are present. On the source surface, in a frame rotating with the Sun at the equatorial rotation rate, we denote the footpoint velocity,

$\mathbf{u}_{\perp}=R_{s s} \omega_{\theta} \hat{e}_{\theta}+R_{s s} \omega_{\phi} \sin \theta \hat{e}_{\phi}$

and assert $\nabla \cdot \mathbf{u}_{\perp}=0$ so that steady state solutions may be found. Notably, we have not placed restrictions on the $(\theta, \phi)$ dependence of $u_{\perp}$. A general solution for the magnetic field in the interaction band (and in the co-rotating frame) is

$$
\begin{aligned}
\mathbf{B}= & B_{s s} \frac{R_{s s}^{2}}{r^{2}}\left[\left(1-\frac{r \omega_{\theta}}{V^{2}} \frac{\partial V}{\partial \theta}\right) \hat{e}_{r}-\frac{r \omega_{\theta}}{V} \hat{e}_{\theta}\right. \\
& \left.-\frac{\left(\Omega_{\odot}+\omega_{\phi}\right) r \sin \theta}{V} \hat{e}_{\phi}\right] .
\end{aligned}
$$

The lower left and right curves in Fig. 2 show the magnetic field structure in FALTS. The left (right) plot shows the configuration for footpoints on one side of the coronal hole that move from inside (outside) to outside (inside) the coronal hole.

Parametric curves for the field lines in the interaction bands are discussed here. These parametric curves describe the radial and azimuthal position $\left(r_{B}, \phi_{B}\right)$ along the field line in the co-rotating frame as a function of co-latitude:

$$
\begin{aligned}
& r_{B}(\theta)=V(\theta) \frac{r_{1}}{V_{1}}-V(\theta) \frac{\theta-\theta_{1}}{\omega_{\theta}} \\
& \phi_{B}(\theta)=\phi_{1}+\left(\Omega_{\odot}+\omega_{\phi}\right) \frac{\theta-\theta_{1}}{\omega_{\theta}} .
\end{aligned}
$$

The initial positions at co-latitude $\theta_{1}$ are $\left(r_{1}, \phi_{1}\right)$. To achieve these solutions, we have assumed that $\omega_{\theta}$ is constant. Thus, the solution provides a reasonable approximation over a limited region of source longitudes $\left(<90^{\circ}\right)$ in the co-rotating frame. We may now solve for the distance $s$ to the termination shock (TS) along a given field line, as shown in Fig. 3. Despite the considerably shorter distances to the TS along FALTS field lines, the angular departure from the more azimuthal Parker spiral is only $\sim 10^{\circ}$.

Figure 3 shows that the field lines in FALTS provide very direct connections to the TS. FALTS then provide energetic particles ready access into the inner heliosphere.

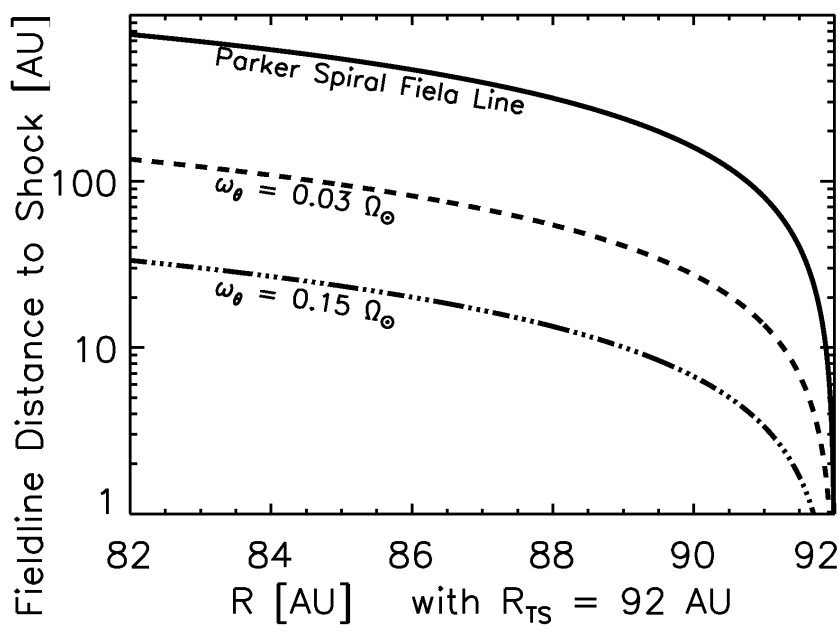

Fig. 3. The distance along a field line to the TS at $35^{\circ}$ latitude. The curves shown are for different values of the parameter $\omega_{\theta}$, which characterizes the angular rate in latitude of footpoint motion on the Sun. Values for all other parameters in equation are unchanged from Fig. 2. The solid curve with $\omega_{\theta}=0$ represents the standard Parker spiral. A value of $\omega_{\theta}=0.15 \Omega \odot$ is of the order of magnitude of differential rotation. The figure shows that even slow footpoint motions on the Sun lead to field lines in the interaction band that provide much more direct connections to the TS than the Parker spiral. If the Voyager 1 spacecraft crossed these FALTS field lines near the TS, it would observe anomalously high intensities of energetic particles. Thus, the FALTS field lines provide one potential explanation for recent Voyager 1 observations.

\section{Conclusions}

We have outlined here newly emerging views concerning the global magnetic configuration of the heliosphere and the reversal of the heliospheric magnetic field, which may be driven largely by coronal mass ejections.

These new concepts for the global structure and reversal of the heliospheric magnetic field may require new models for the propagation of energetic particles and cosmic rays. FALTS provide ready access for energetic particles to the inner heliosphere. The CME model for the heliospheric magnetic field reversal provides an inherent link between the release of CMEs and the global evolution of the heliospheric magnetic field.

A goal of International Heliophysical Year activities is to understand the detailed links between magnetic field evolution, cosmic ray evolution and energetic particle evolution in different regimes in the heliosphere. Here, we have illustrated the implications of open magnetic flux conservation, causing an inherent connection between the footpoint motions back at the Sun, the global structure of solar wind, the release of coronal mass ejections and the global structure and evolution of the heliospheric magnetic field. An important next step is to build these effects into global energetic particle and cosmic ray models of the heliosphere. The magnetic field 
models we have outlined provide an interconnected view of solar and heliospheric evolution.

Acknowledgements. This research was supported by the National Science Foundation under Agreement ATM-012950, which funds the CISM project of the STC program. N.C. was additionally funded by NASA grant NNG06GC18G. NAS was supported by the NASA's Earth-Moon-Mars Radiation Environment Modeling (EMMREM) Project and NASA's Interstellar Boundary Explorer Project.

Edited by: V. V. Izmodenov

Reviewed by: R. Forsyth and C. Smith

\section{References}

Arge, C. N., Hildner, E., Pizzo, V. J., and Harvey, J. W.: Two solar cycles of nonincreasing magnetic flux, J. of Geophys. Res., 107, 1319-1327, doi:10.1029/2001JA000503, 2002.

Babcock, H. W.: The topology of the Sun's magnetic field and the 22-year cycle, Astrophys. J., 133, 572-577, 1961.

Bieber, J. W. and Rust, D. M.: The Escape of Magnetic Flux from the Sun, Astrophys. J., 453, 911-918, doi:10.1086/176451, 1995.

Bird, M. K. and Edenhofer, P.: in: Physics of the Inner Heliosphere, edited by Schwenn, R. and Marsch, E., vol. 1, chap. 13, Springer Verlag, Berlin, 1990.

Bothmer, V. and Rust, D. M.: The Field Configuration of Magnetic Clouds and the Solar Cycle, in: Coronal Mass Ejections, edited by Crooker, N., Jocelyn, J. A., and Feynman, J., vol. 99, Geophysical Monograph, American Geophysical Union, 139-146, 1997.

Bothmer, V. and Schwenn, R.: The Structure and Origin of Magnetic Clouds in the Solar Wind, Ann. Geophys., 16, 1-24, 1998.

Burlaga, L. F.: Interplanetary Stream Interfaces, J. Geophys. Res., 79, 3717-3728, 1974.

Crooker, N. U., Gosling, J. T., and Kahler, S. W.: Reducing heliospheric magnetic flux from coronal mass ejections without disconnection, J. of Geophys. Res., 107, 3-1, 2002.

Crooker, N. U., Forsyth, R., Rees, A., Gosling, J. T., and Kahler, S. W.: Counterstreaming electrons in magnetic clouds near 5 AU, J. of Geophys. Res., 109, 6110-6121, doi:10.1029/ 2004JA010426, 2004.

Fisk, L. A.: Motion of the footpoints of heliospheric magnetic field lines at the Sun: Implications for recurrent energetic particle events at high heliographic lattitudes, J. Geophys. Res., 101, 15 547-15 553, 1996.

Fisk, L. A. and Schwadron, N. A.: The behavior of the open magnetic field of the Sun, Astrophys. J., 560, 425-431, 2001.

Fisk, L. A., Zurbuchen, T. H., and Schwadron, N. A.: On the coronal magnetic field: consequences of large-scale motions, Astrophys. J., 521, 868-874, 1999.

Gopalswamy, N., Lara, A., Yashiro, S., and Howard, R. A.: Coronal Mass Ejections and Solar Polarity Reversal, Astrophys. J. Lett., 598, L63-L66, doi:10.1086/380430, 2003.

Gosling, J. T., Asbridge, J. R., Bame, S. J., and Feldman, W. C.: Solar Wind Stream Interfaces, J. Geophys. Res., 83, 1401-1412, 1978.
Gosling, J. T., Baker, D. N., Bame, S. J., Feldman, W. C., and Zwickl, R. D.: Bidirectional solar wind heat flux events, J. Geophys. Res., 92, 8519-8535, 1987.

Hale, G. E. and Nicholson, S. B.: The Law of Sun-Spot Polarity, Astrophys. J., 62, 270-276, doi:10.1086/142933, 1925.

Hundhausen, A. J. and Burlaga, L. F.: A model for the origin of solar wind stream interfaces, J. Geophys. Res., 80, 1845-1848, 1975.

Jones, G. H., Balogh, A., and Smith, E. J.: Solar magnetic field reversal as seen at Ulysses, Geophys. Res. Lett., 30, 2-1, 2003.

Krimigis, S. M., Decker, R. B., Hill, M. E., Armstrong, T. P., Gloeckler, G., Hamilton, D. C., Lanzerotti, L. J., and Roelof, E. C.: Evidence that Voyager-1 exited the Solar Wind at $\sim 85$ AU, Nature, 426, 45-52, 2003.

Leighton, R. B.: A Magneto-Kinematic Model of the Solar Cycle, Astrophys. J., 156, 1-11, 1969.

Low, B. C.: Global magnetic-field reversal in the corona, in: Solar varibility and its effects on climate, edited by Pap, J. and Fox, P., 141, 51-64, 2004.

Lynch, B. J., Gruesbeck, J. R., Zurbuchen, T. H., and Antiochos, S. K.: Solar cycle dependent helicity transport by magnetic clouds, J. Geophys. Res., 110, 8107-8115, doi:10.1029/ 2005JA011137, 2005.

McComas, D. J., Barraclough, B. L., Funsten, H. O., Gosling, J. T., Santiago-Muñoz, E., Skoug, R. M., Goldstein, B. E., Neugebauer, M., Riley, P., and Balogh, A.: Solar wind observations over Ulysses' first full polar orbit, J. Geophys. Res., 105, 10419 10434, 2000a.

Nash, A. G., Sheeley, Jr., N. R., and Wang, Y.-M.: Mechanisms for the rigid rotation of coronal holes, Solar Phys., 117, 359-389, 1988.

Owens, M. J. and Crooker, N. U.: Coronal mass ejections and magnetic flux buildup in the heliosphere, J. of Geophys. Res., 111, 10 104, doi:10.1029/2006JA011641, 2006.

Owens, M. J., Schwadron, N. A., Crooker, N. U., Hughes, W. J., and Spence, H. E.: Role of coronal mass ejections in the heliospheric Hale cycle, Geophys. Res. Lett., 34, 6104, doi:10.1029/ 2006GL028795, 2007.

Parker, E. N.: Dynamics of the Interplanetary Gas and Magnetic Fields, Astrophys. J., 128, 664-676, 1958.

Pizzo, V. J.: The evolution of corotating stream fronts near the ecliptic plane in the inner solar system. I - Two-dimensional fronts, J. Geophys. Res., 94, 8673-8684, 1989.

Rees, A. and Forsyth, R. J.: Magnetic clouds with east/west orientated axes observed by Ulysses during solar cycle 23, Geophys. Res. Lett., 30, 4-1, 2003.

Riley, P., Gosling, J. T., and Crooker, N. U.: Ulysses Observations of the Magnetic Connectivity between Coronal Mass Ejections and the Sun, Astrophys. J., 608, 1100-1105, doi: 10.1086/420811, 2004.

Schatten, K. H., Wilcox, J. M., and Ness, N. F.: A model of interplanetary and coronal magnetic fields, Solar Phys., 6, 442-455, 1969.

Schrijver, C. J., DeRosa, M. L., and Title, A. M.: What Is Missing from Our Understanding of Long-Term Solar and Heliospheric Activity?, Astrophys. J., 577, 1006-1012, doi:10.1086/342247, 2002.

Schwadron, N. A. and McComas, D. J.: Heliospheric "FALTS" Favored Acceleration Locations at the Termination Shock, Geo- 
phys. Res. Lett., 30, 1587, doi:10.1029/2002GL016\,499, 2003.

Siscoe, G. L.: Three-dimensional aspects of interplanetary shock waves, J. Geophys. Res., 81, 6235-6241, 1976.

Smith, C. W. and Phillips, J. L.: The role of coronal mass ejections and interplanetary shocks in interplanetary magnetic field statistics and solar magnetic flux ejection, J. Geophys. Res., 102, 249-262, doi:10.1029/96JA02678, 1997.

Smith, E. J.: Magnetic fields throughout the heliosphere, Adv. in Space Res., 13, 5-14, 1993.

Smith, E. J. and Balogh, A.: Open Magnetic Flux: Variation with Latitude and Solar Cycle, in: Solar Wind Ten, edited by Velli, M., Bruno, R., Malara, F., and Bucci, B., 679 American Institute of Physics Conference Series, 67-70, 2003.

Smith, E. J., Balogh, A., Forsyth, R. F., Tsurutani, B. T., and Lepping, R. P.: Recent Observations of the Heliospheric Magnetic Field at Ulysses: Return to Low Latitude, Adv. in Space Res., 26, 823-832, 2000.
Snodgrass, H. B.: Magnetic Rotation of the solar photosphere, Astrophys. J., 270, 228-231, 1983.

Wang, Y.-M. and Sheeley, N. R.: The Solar Wind and Its Magnetic Sources at Sunspot Maximum, Astrophys. J., 587, 818822, 2003.

Wang, Y.-M., Sheeley, N. R., and Andrews, M. D.: Polarity reversal of the solar magnetic field during cycle 23, J. of Geophys. Res., 107, 1465-1471, doi:10.1029/2002JA009463, 2002.

Yashiro, S., Gopalswamy, N., Michalek, G., St. Cyr, O. C., Plunkett, S. P., Rich, N. B., and Howard, R. A.: A catalog of white light coronal mass ejections observed by the SOHO spacecraft, J. of Geophys. Res., 109, 7105-7112, doi:10.1029/2003JA010282, 2004. 